\title{
Análisis de la
}

construcción lo

que es. Hacia una

Gramática de la

Aplicación

Sofía Gutiérrez Böhmer

Recebido em: 28 de jultho de 2019

Aceito em: 24 de setembro 2019
Licenciada en Letras por la

Universidad de Buenos Aires y miembro del Proyecto UBACyT a cargo de la Dra. Claudia Borzi. Codirige el Programa de Enseñanza de Español a Inmigrantes Adultos Trabajadores de la Economía Popular dependiente de la Secretaría de Extensión Universitaria y Bienestar Estudiantil de la Universidad de Buenos Aires.

Contato: sofia.bohmer@gmail. com

Argentina 


\section{PALABRAS CLAVE: marcadores discursivos; perírasis de relativo; 10 que es; Gramática de la Aplicación}

KEYWORDS: discourse markers; cleft sentences; lo que es; Grammar of Application
Resumen: El presente trabajo tiene por objetivo analizar el comportamiento de la construcción lo que es en casos como "pero lo que es tablados y Teatro de Verano, es muy poca gente la que sale". Al momento de consultar la literatura sobre el tema, se ha encontrado un gran consenso en que dicha estructura es una perífrasis de relativo. Sin embargo, el análisis revela que lo que es se comporta como un marcador discursivo de concreción/ejemplificación. Si bien esta última hipótesis comparte muchos atributos con la de la perírrasis, entender que lo que es es un marcador describe, aunque de manera teórica, más adecuadamente el fenómeno para su ulterior aplicación en la enseñanza de Español como Lengua Extranjera. Se ha repasado de manera crítica la literatura sobre el tema y se han expuesto argumentos que defienden la inclusión de lo que es en la catesoría de los marcadores discursivos en general y de los marcadores de ejemplificación/concreción en particular.

Abstract: The present work aims to analyze the behavior of lo que es in cases such as "pero lo que es tablados y Teatro de Verano, es muy poca gente la que sale". At the time of consulting the literature on the subject, a general consensus reports that it is a cleft construction. However, the analysis here presented revealed that the construction functions as an exemplifying discourse marker. Despite the fact that both hypotheses (cleft construction and discourse marker) have many attributes in common, understanding lo que es as a marker describes the structure more adequately, albeit theoretically, for its further application in Spanish teaching as a Foreign Language. Literature on the subject has been critically reviewed and arguments have been presented defending the inclusion of lo que es in the category of discourse markers in general and exemplifying discourse markers in particular. 


\section{INTRODUCCIÓN}

A menudo surge una brecha metodológica autogenerada por los límites mismos de las disciplinas involucradas en la Enseñanza de Segundas Lenguas (ESL), a saber, la brecha entre pedagogos que no hacen teoría y teóricos sin bajada práctica. Martín Sánchez, por caso, reflexiona sobre la elección del enfoque teórico-gramatical más adecuado para la enseñanza y dice que "el profesor debe buscar información y trabajar con las diferentes gramáticas que existen: tradicional, comparada, estructural, generativa, histórica o descriptiva” (Martín Sánchez, 2010, 61). Por otro lado, Castañeda Castro y Melguizo Moreno toman, para una aproximación pedagógica a los principales usos del clítico se, una postura fuerte por la Gramática Cognitiva partiendo, empero, de clasificaciones hechas por autores, muchos de los cuales no pertenecen al enfoque elegido o, incluso, al mundo de ESL (Castañeda Castro y Melguizo Moreno, 2006).

A su manera, los dos estudios han partido de la premisa de que cualquier trabajo teórico será igualmente útil para su aplicación práctica en el terreno de la clase, siempre que haya una mediación pedagógica: en Martín Sánchez, un profesional ELE debe trabajar con cualquier enfoque teórico; para Castañeda Castro y Melguizo Moreno, una propuesta pedagógica con una fuerte preferencia por un enfoque teórico puede armarse sobre categorizaciones hechas por otros enfoques.

Esta brecha metodológica termina favoreciendo que los resultados de un estudio teórico cualquiera sean considerados válidos de una vez y para todos los casos gracias a la sola aplicación del método científico. Esta 
confianza absoluta en la rigurosidad de un método es lo que, en El oficio de sociólogo, Bourdieu, Chamboredon y Passeron llamaron "rigorismo tecnológico" (Bourdieu, Chamboredon y Passeron (2002 [1975]), 21); para combatirlo, los autores invitan a sostener una actitud crítica en todas las etapas del proceso de investigación (la elección del tema, la construcción del objeto de estudio, las técnicas de recolección de datos, el análisis de los datos, etc.).

Algunos años después, ya en el campo de la Gramática y de la Adquisición de Segundas Lenguas, Corder sostuvo que no existe una sola gramática observacionalmente adecuada (Corder, 1982 [1981], 59), pero sí existen criterios para considerar una gramática como descriptivamente adecuada. Esta propuesta es complementaria a aquella de El oficio de sociólogo: si la rigurosidad del método empleado para un estudio teórico no garantiza la validez de sus resultados siempre y para cualquier situación, mucho menos lo hará la mera "observabilidad" del fenómeno bajo análisis; en otras palabras, una descripción gramatical empírica no será automáticamente la descripción más aplicable. Necesitamos, por tanto, dar con la gramática que sea descriptivamente más adecuada para la práctica ESL y, para ello, la vigilancia crítica del proceso teórico deberá estar puesta al servicio de ESL desde el comienzo.

Este trabajo se propone analizar el comportamiento de la construcción lo que es en casos como (1a), poniendo especial vigilancia crítica en que su descripción sea, si bien teórica, adecuada para su ulterior aplicación en ELE. 
(1)

a. [La metafísica] es una de las cosas que aprendí / lo que más me gusta ¿viste? / es lo que es mi cable a tierra [MONV_M22_022] ${ }^{1}$

b. La metafísica es una de las cosas que aprendí, lo que más me gusta ¿viste? Es mi cable a tierra.

Se ha elegido esta construcción, por ser de uso puntual y poco investigada y permitir, por tanto, un desarrollo razonablemente acabado dentro de los límites de esta publicación.

Al momento de buscar respuestas, la reducida literatura teórica se mueve en dos direcciones: o bien desarrolla el fenómeno de manera muy sucinta, haciendo solo una breve mención de su significado y uso (Carbonero Cano, 2001; Pérez Álvarez, 2014), o bien lo desarrolla de manera intrincada (Lastra y Butragueño, 2016), volviendo difícil una adaptación pedagógica. Me referiré a la literatura en el siguiente apartado. Por el momento, baste con mencionar que existe un consenso con respecto a que la construcción se trata de una perífrasis de relativo (Es Juan el que viene / El que viene es Juan / Juan es el que viene) ${ }^{2}$. Por otro lado, desde la corrección de estilo se

1 Los ejemplos, salvo especificación contraria, pertenecen al Corpus PRESEEA-Montevideo (2007), Proyecto para el Estudio Sociolingüístico del Espańol de Espańa y América <http://www.linguas. net/preseea>.

2 Esta estructura ha sido también llamada estructura de realce, copulativa enfática de relativo, construcción oforma de relieve y oración hendida. Para los fines de este trabajo, dichas nominaciones son permutables. 
ha mencionado que su ausencia no afecta las condiciones de verdad (1b), razón por la cual se desalienta su uso ${ }^{3}$.

Para una primera aproximación al significado inicialmente opaco de la construcción, he utilizado el recurso de la paráfrasis. Al hacerlo, he encontrado que lo que es puede ser reemplazado sin mayor dificultad con por ejemplo (2). Al mismo tiempo, hay situaciones en las que el reemplazo parece funcionar mejor con en lo que respecta a, como es el caso de (3) ${ }^{4}$; aún así, seguiré incluyéndolo dentro del mismo grupo. Daré las razones de esta inclusión en el apartado 4.

(2)

a. y planificás lo que vas a hacer / las escenas de la la parte de obra / eh I lo que es canciones / música / coros y todo [MONV_H11_035]

b. y planificás lo que vas a hacer / las escenas de la la parte de obra / eh / por ejemplo canciones / música / coros y todo

(3)

a. pero lo que es / tablados y Teatro de Verano / es muy poca gente la que sale [MONV_H11_035]

3 Un ejemplo de ello se puede encontrar en el sitio Fundéu BBVA (2014).

4 Para facilitar la lectura de los ejemplos tomados del corpus, se ha eliminado la mayoría de las marcas de transcripción, salvo en aquellos casos relevantes. En todos los casos, se ha mantenido la ortografía original. La cursiva pertenece a la autora. 
b. pero en lo que respecta a / tablados y Teatro de Verano / es muy poca gente la que sale

Así, si lo que es puede ser parafraseado con por ejemplo, debo postular la hipótesis de que la construcción funciona como un marcador discursivo (MD), más específicamente de concreción o ejemplificación.

Defenderé, pues, la hipótesis del MD y, para ello, recorreré de manera crítica la literatura consultada hasta la fecha y la clasificación de la forma como perífrasis de relativo (PdR); detallaré, luego, las características atribuidas a los MDs y la pertinencia de estas a la hora de describir lo que es; en tercer lugar, ahondaré en las especificidades de los marcadores de concreción/ejemplificación, compartidas por lo que es, para determinar su función.

\section{Lo que es no es UnA perírasis de relativo}

Desafortunadamente, la literatura que analiza esta construcción del español no es numerosa y la que he logrado reunir, en su mayoría, hace una mención fugaz a la estructura dentro de un estudio mayor sobre otras construcciones con lo que.

Pedro Carbonero Cano menciona brevemente esta construcción y la considera como una forma de relieve (otra denominación para las PdR) que contrasta a veces "unos elementos del discurso en relación con otros" $(2001,89)$.

5 En el apartado 2., se hará mención a presencia de concordancia o falta de ella en este tipo de construcciones (lo que es Tablados y Teatro de Verano vs lo que son Tablados y Teatro de Verano). 
Pérez Álvarez, a su vez, también sostiene que lo que es es una oración escindida o hendida (PdR), en un estudio más general sobre las funciones oracionales y discursivas de lo que. Este texto se centra en lo que viene siendo, que describe como "una fórmula utilizada mientras se planea el discurso subsecuente" (2014, 91). Sin embargo, no desarrolla otras formas de lo que + ser.

El trabajo más detallado y con el que discutiré en adelante le pertenece a Lastra y Butragueńo (2016). Los autores definen lo que es como un tipo de PdR.

Las PdR, de acuerdo a Moreno Cabrera, son un proceso de expansión perifrástica que denota el mismo tipo de suceso que la estructura contraída (Moreno Cabrera, 1999, 4250):

\section{(4)}

a. Juan ha llegado tarde.

b. Es Juan \{quien/el que\} ha llegado tarde.

c. (Íbid, 4247)

Estas perífrasis en español presentan 3 variantes (Íbid, 4251):

Cuadro 1. Variantes de las PdR según Moreno Cabrera (1999)

\begin{tabular}{|l|l|l|}
\hline PdR de relativa libre (RL) & \multicolumn{1}{|c|}{$\begin{array}{c}\text { PdR de constituyente } \\
\text { escindido (CES) }\end{array}$} & \multicolumn{1}{|c|}{ PdR de cópula (COP) } \\
\hline $\begin{array}{l}\text { Tienen la RL en primer } \\
\text { lugar. Ejemplo: } \\
\text { El que viene es Juan. }\end{array}$ & $\begin{array}{l}\text { Presentan el CES en primer } \\
\text { lugar. Ejemplo: } \\
\text { Juan es el que viene. }\end{array}$ & $\begin{array}{l}\text { Presentan la cópula en } \\
\text { primer lugar. } \\
\text { Ejemplo: } \\
\text { Es Juan el que viene. }\end{array}$ \\
\hline
\end{tabular}


Para Lastra y Butragueño, la especificidad de lo que es es la de ser un marcador de prominencia informativa que han dado en llamar perifrasis informativa de relativo (PIR) y cuya función es "revelar la existencia de un constituyente oracional o de un orientador extraoracional" (Lastra y Butragueño, 2016, 271). De manera más simple, el consecuente en las PIR siempre refiere al foco (Íbid, 272) ${ }^{6}$.

Las características propuestas para las PIR, que pueden ser compartidas por otras construcciones con lo que, son: (a) la invariabilidad de lo que; (b) el carácter objetival del correferente; (c) la naturaleza referencial de lo; (d) la relación predicativa entre lo y el correferente; (e) la explicitud y posición consecuente del correferente, así como su dominio oracional y carácter de frase nominal; (f) la función informativa desempeñada por el consecuente; $(\mathrm{g}$ ) la concordancia no categórica entre el verbo débil y el consecuente; (h) el carácter no extraíble del consecuente; (i) la tendencia al presente en el verbo débil y la improbabilidad de que reciba prominencia prosódica; (j) la existencia de PIR prototípicas y PIR periféricas; (k) la modulación de la fuerza ilocutiva por medio de algunos casos de PIR; (l) la naturaleza no epistémica, no evidencial y habitualmente no contrastiva de las PIR; (m) el papel de la cuantificación y de la negación; y (n) el carácter comúnmente aseverativo de los actos de habla en los que se instalan las PIR.

6 Estos autores entienden por foco aquella parte de la oración que proporciona información nueva, aunque también puede definirse como la aportación relevante o como la selección entre varias opciones posibles. 
Como se detallará en breve, comparto con los autores un gran número de los rasgos señalados para la construcción; mi trabajo en esta sección será demostrar que estos rasgos contradicen la hipótesis de la PdR y dejan la puerta abierta para la interpretación como MD.

Veamos, pues, el primero de los rasgos expuestos: la invariabilidad de $l o$ que (como se especifica en el inciso [a]). La razón por la que esta característica debilita la clasificación de las PIR como una PdR es que estas últimas sí suelen presentar concordancia de género y número entre el pronombre relativo y su correferente.

[La pizzería Montecarlo] es la que está antes de Calcagno $\left[\mathrm{MONV}_{-}\right.$ M12_020]

En (b), se habla del carácter objetival del consecuente, ya que las PIR suelen referirse a objetos y no a seres animados. Las PdR, por otro lado, no manifiestan esta tendencia, como se ilustra en (4).

Más tarde, se establece la posición consecuente del correferente, referida en (e), que es coherente con la idea expresada en (h), a saber, que el correferente no es extraíble. En otras palabras, ambos puntos sostienen que la construcción no participa de las 3 variantes propuestas por Moreno Cabrera (1999) para las PdR en español. También en (h), al analizar el carácter no extraíble, los autores mencionan que 
tener que ver con parece funcionar exactamente igual que una PIR: la perífrasis es prescindible, el consecuente no personal no es extraíble, se respeta y potencia su función informativa, se ofrece una lectura absoluta de entidad, etc. (Lastra y Butragueño, 2016, 277)

También se menciona la equivalencia de algunas PIR con lo que se llamal lo que llaman y lo que se refiere a. Esta característica aleja a la construcción de las PdR, puesto que las últimas no establecen estas relaciones de equivalencia (6). De hecho, ello da cuenta de una cierta semanticidad de la construcción que las PdR no poseen.

(6)

a. Lo que es Juan es alto [PdR].

b. ${ }^{*}$ Lo que tiene que ver con Juan es alto.

El quinto problema es la concordancia no categórica entre el verbo débil y el consecuente, expuesta en (g). Si bien lo que es no presenta una gramaticalización absoluta, tiene una tendencia a aparecer en tercera persona del singular generando frases en las que no hay concordancia con el correferente como en (7). Este caso, además, expone una falta de concordancia doble: como construcción sujetiva con el verbo de la cláusula principal y hacia adentro de la presunta cláusula sustantiva con el correferente ([7a] y [7b]). 
(7)

a. Preciosas Ana!!!!!!! Espero que tus chicos las usen, porque lo que es los míos, ni la han estrenado. Feliz semana. ${ }^{7}$

b. mi animal favorito son los felinos no hay un animal predeterminado que me guste pero me encantan lo que es los gatos, leones, tigres, pumas, etc... ${ }^{8}$

Esta falta de concordancia dura es difícil de imaginar en una PdR:

(8)

a. *Lo que ni la han estrenado es los míos.

b. *Lo que me encantan es los gatos, leones, tigres, pumas.

En sexto lugar, las PdR tampoco presentan la tendencia al presente de las PIR (inciso [i]). El tiempo de la cópula en las PdR sí puede ser "coincidente con el momento de emisión", el presente, pero también hay "concordancia temporal de la cópula con el verbo de la oración principal expandida” (Moreno Cabrera, 1999, 4289-4290).

En (m), los autores desarrollan la posibilidad de cuantificación en las PIR. Obsérvese (9).

7 Sánchez Yánez, María José. 25 de abril de 2016. [Comentario en un blog]. Recuperado de <http:// patchworkymanualidadesdeana.blogspot.com.ar/2016/04/bolsas-para-la-ropa-interior.html>

8 Valenzuela, Andrea. 11 de mayo de 2015. [Comentario en un blog]. Recuperado de <http:// los-mejores-trucos-de-stardoll-dani.blogspot.com.ar/2015/05/preguntas-respuestas-muybuenas.html> 
(9)

entonces yo sola me fui/ caminando/ todo lo que es la Lagunilla [ME293-13M-07, turno 179]. (Lastra y Butragueńo, 2016, 283)

Una cuantificación similar en una PdR es insostenible dado que genera, o bien emisiones inaceptables, o bien la estructura deja de ser una PdR para ser una construcción copulativa no expansiva. Obsérvense los ejemplos en (10).

a. *Toda la que vino fue la gente.

b. Todo lo que tomé ayer fue un café [*PdR/equivalente a "solo tomé un café"]

En octavo lugar, estos autores describen la dispersión social de la forma y encuentran que, si bien la variable sociolingüística "nivel de estudios" no es significativa, sí lo son las de género y edad. Sin embargo, sería esperable que las PdR presentaran una distribución en términos de registro o distribución de la información, pero no una distribución sociolingüística.

Finalmente, hay una diferencia clave entre las PIR y las PdR a la que no se ha atendido: el hecho de que ninguna de las variantes de una PdR propone el verbo cópula ser inmediatamente después del relativo. Esta secuencia en efecto ocurre en las PdR, pero solo cuando el elemento escindido es un atributo. Véase (11). 
(11)

a. Lo que es Juan es alto

b. Juan es alto

Se puede ver en (11) que el verbo ser, adyacente a lo que, no es parte de la estructura expandida, sino de la versión contraída (11b). Al mismo tiempo, esta combinación no es privativa de las PdR; cualquier cláusula subordinada sustantiva en la que el relativo cumple la función de predicativo sujetivo puede presentar esta estructura.

se educó muy bien / ella / la verdad que / bueno se casó tiene hijos y nietos y / qué sé yo y este / está bien / eh salió bien la gurisa / para / dentro de lo que es esta sociedad ¿̨no? [MONV_M32_032]

En suma, 9 de las 14 características expuestas para las PIR contradicen la hipótesis de la PdR. Ante una situación similar, Cifuentes Honrubia refiere chistosamente al comentario de una compañera de trabajo: Si tenemos una cabra que no cumple ninguno de los requisitos de las cabras, no es una cabra, es una vaca (Cifuentes Honrubia, 1999, 46). 


\section{Lo QUE ES UN MD}

Si lo que es no es una PdR, retomando el apartado anterior, de esto no se desprende automáticamente que sea un MD. En ese apartado me propongo justificar la inclusión de lo que es en esta categoría.

Para comenzar, Martín Zorraquino y Portóles Lázaro han definido a los MDs como

unidades lingüísticas invariables [que] no ejercen una función sintáctica en el marco de la predicación oracional -son pues, elementos marginales- $y$ poseen un cometido coincidente en el discurso: el de guiar, de acuerdo con sus distintas propiedades morfosintácticas, semánticas y pragmáticas, las inferencias que se realizan en la comunicación $(1999,4057)$.

Veamos cómo se ajusta lo que es a esta definición.

\subsection{Invariabilidad}

Los MD proceden de la evolución de una serie de sintagmas que perdieron sus posibilidades flexivas, combinatorias y su significado conceptual. Por ello, son unidades invariables. Se admite, no obstante, que no todos los MDs están igualmente gramaticalizados (Íbid, 4060).

En el caso de lo que es, la tendencia al presente y la concordancia no categórica referida podría ser indicio de que esta construcción está en proceso de gramaticalización. Este asunto deberá ser respaldado por un estudio diacrónico pero, en el plano de lo sincrónico, he dado con un ejemplo como (13). 
DAT // es un eh tipo un coso que te ayuda a ir progresando a lo que es el tema de obra / a lo que nosotros no somos obreros [MONV_H11_035]

Este fragmento contiene una secuencia yuxtapuesta con la que el informante detalla la ayuda que el DAT (Departamento de Apoyo Técnico) le ofrece (a ir progresando, a lo que es el tema de obra, a lo que nosotros no somos obreros). Como se puede observar, en el tercer miembro de la secuencia, la construcción pierde su elemento flexivo, volviéndola aún más invariable y permitiendo que anteceda a una cláusula. Este ejemplo es único; aún así, es un comportamiento que más difícilmente se da cuando la dependencia es de nivel oracional.

Otro posible indicio de gramaticalización es la existencia de casos de categorización dudosa como (14).

a. y empezás a, yo por ahí ipha! empezás a como a pensar en todo lo que fue tu vida con él ¿̨no? // este, igual me acuerdo que tampoco quise pensar demasiado ¿̨no? [MONV_H12_006]

b. y empezás a, yo por ahí ¡pha! empezás a como a pensar en como fue tu vida con él ¿̨no?

Aquí, el informante está refiriendo a las características de la vida con su padre, por lo que la construcción lo que fue funciona de manera atributiva. 
Nuevamente, se trata de un ejemplo único pero resultará interesante atender a este tipo de situaciones en un corpus ampliado.

\subsection{Ausencia de función sintáctica}

Lastra y Butragueño (2016) han defendido, en el inciso (e), que el dominio de esta construcción es oracional. Sin embargo, se observa en los ejemplos (15) - (18) un salto hacia el discurso.

pero lo que es / tablados y Teatro de Verano / es muy poca gente la que sale [MONV_H11_035]

de hecho ahora / ahora están haciendo todo un tema de la sanitaria probablemente te hayas enterado / todo lo que es zona Cerro / capaz que escuchaste no sé [MONV_H21_043]

o cualquier cosa / y estábamos hasta / lo que era verano hasta las doce de la noche [MONV_H21_043]

yo te empiezo a decir / bueno / cerramos los ojos / nos aflojamos / sentimos una luz / que nos invade / sí / que entre por nuestro <ininteligible/> se abre 
y entre esa luz y lo ilumina [...] todo lo que es la mente / entonces claro / tú estás atenta a aquello que yo te voy diciendo [MONV_M22_022]

Tanto en (15), ya mencionado en (3), como en (16) y (17), se puede observar que el consecuente introducido por la construcción no constituye un actante de los sucesos que narra, ya que carece de la preposición en que los introduciría como actantes temporales o espaciales. (18) es un caso aún más interesante dado que la construcción y su consecuente no son actantes de la cláusula precedente ni de la subsecuente.

Sumado a lo anterior, Martín Zorraquino y Portolés Lázaro (1999) han trazado, además, una serie de propiedades relacionadas a la movilidad, la independencia prosódica, los modificadores, los complementos, la coordinación, la negación y la PdR.

\subsection{Movilidad}

Los MD, al no estar integrados en la oración, pueden con frecuencia aparecer en diferentes posiciones. La movilidad, no obstante, tiene sus restricciones. Un marcador no se puede situar entre el núcleo y sus adyacentes específicos, por ejemplo. Asimismo, existen MDs que casi siempre aparecen encabezando su miembro discursivo $(1999,4063)$. Esto es lo que sucede con lo que es, el cual, a su vez, presenta una tendencia a ocurrir en posición prenominal. Esta posición prenominal también es una tendencia de otros marcadores de concreción, como se mencionará en el apartado 5. 


\title{
3.4. Independencia prosódica
}

En general, los MDs "se encuentran limitados [...] por la entonación", según lo entienden Martín Zorraquino y Portolés Lázaro (1999, 4064). Aún así, un trabajo de Fernández Bernárdez (1994-1995), comentado por Martín Zorraquino y Portolés Lázaro en el texto citado, encuentra que los marcadores de ejemplificación suelen presentarse como unidades fónicas independientes, pero no se comportan de manera homogénea. Sostiene que:

\begin{abstract}
Algunos siempre lo son [independientes]. Otros, pueden serlo o no, sin que este rasgo afecte en general a su significado [...]. No obstante, existen casos en que el hecho de que el marcador por ejemplo vaya entre pausas, o no, es pertinente. Además, aunque no se confirme en todos los casos, el marcador como puede variar de significado si va precedido de pausa o no (Fernández Bernárdez, 1994-1995, 140).
\end{abstract}

Si esto no ha impedido la incorporación de los marcadores de ejemplificación como MD, no veo por qué debería impedir la incorporación de lo que es.

Adicionalmente, he hallado en mi cuerpo de datos tres casos en los cuales lo que es presenta algún tipo de suspensión que despega a la construcción del resto de la frase y ninguna PdR que se comporte del mismo modo. Véase (3) más arriba, donde se observa una pausa breve después de la construcción, y (19) y (20) a continuación.

yo estoy en comisión de obra que se encarga de<alargamiento/ $>$ de todo lo que e<alargamiento/>s los gas < palabra_cortada/ $>$ la la parte de de<alargamiento/> o<alargamiento/>bra [MONV_H11_035] 
(20)

que sé yo ¿̨no? / no le doy mucho tiempo para mis cosas / por ejemplo yo soy una persona muy espiritua<alargamiento/>l entonces / me gusta mucho lo que es eh / música / meditar [MONV_M22_022]

En suma, lo que es no se comporta de manera diferente a aquellos marcadores de ejemplificación, que pueden ser unidades fónicas independientes o no.

\subsection{Modificadores, complementos, coordinación, negación y PdR}

Los MDs no reciben, en líneas generales, especificadores y adyacentes complementarios. Tampoco se pueden coordinar entre sí, a menos que se sitúen en inciso. Por último, no pueden ser negados ni se pueden destacar por medio de una PdR. Estas cuatro condiciones se cumplen con lo que es:

(21)

a. ${ }^{*}$ Lo que es de pronto la casa se llovió toda.

b. *Lo que es y además la casa se llovió toda.

c. *No lo que es la casa se llovió toda.

d. *Es lo que es lo que se llovió toda la casa

Martín Zorraquino y Portolés Lázaro han aceptado que mirá se comporta como una partícula discursiva y que se ajusta, en general, a las propiedades establecidas: 
No admiten ningún tipo de complementación $\left({ }^{*}\right.$ mira esto; ${ }^{*}$ mira alli; ${ }^{*}$ mira de reojo, etc.) y solo esporádicamente comparecen con el sujeto (mira tú; mirá vos; mire usted, etc.) o con un vocativo que se refiere a este (mira, Ana; mire, don Pedro, etc.); tampoco admiten la negación (el tipo de construcción no mires, no miréis, etc., no representa al marcador), etc. En cambio, estas partículas pueden muy bien ser moduladas por medio de la entonación $(1999,4181)$.

Aunque las pruebas aplicadas no han sido exactamente las mismas, lo que es también se ajusta de manera general a las propiedades y debería incluirse como MD.

\section{LO QUE ES CUMPLE LA FUNCIÓN DE UN MD DE CONCRECIÓN/EJEMPLIFICACIÓN}

Rechazada la clasificación de lo que es como PdR y defendida su inclusión como MD, en este apartado retomaré la idea de que la construcción lo que es puede ser parafraseada mediante por ejemplo, entre otros. La posibilidad de paráfrasis sugiere, pues, que la construcción equivale a lo que Martín Zorraquino y Portolés Lázaro llamaron operadores de concreción y que, según estos autores, "presentan el miembro del discurso que los incluye como una concreción o ejemplo de una expresión más general” (1999, 4142). Un ejemplo de lo que es funcionando de esta manera es (22[2]), previamente referido, y (23) a continuación.

$(22[2])$

y planificás lo que vas a hacer / las escenas de la la parte de obra / eh / lo que es canciones / música / coros y todo [MONV_H11_035] 
a. por ejemplo lubolos se caracteriza más por / lo que es la tradición / negra / lo que es el gramillero lo que es escobero todas esas cosas [MONV_H11_035]

b. por ejemplo lubolos se caracteriza más por / lo que es la tradición / negra / por ejemplo el gramillero, por ejemplo escobero todas esas cosas

En (22[2]) “canciones, música, coros y todo", secuencia introducida por lo que es, son ejemplificaciones de "la parte de obra". En (23) se observa lo mismo con "gramillero" y "escobero", que son ejemplificaciones de la tradición negra.

También se ha mencionado previamente el hecho de que la construcción puede ser reemplazada por formas como en lo que respecta a, etc., función topicalizadora a la que Martín Zorraquino y Portolés Lázaro no hacen referencia. Errázuriz Cruz, por su parte, los clasifica como marcadores con valor de inicio porque "manifiestan una visión prospectiva y de apertura hacia el acto enunciativo que introducen; controlan el comienzo de un acto discursivo; corresponden a enlaces con valor de topicalización” (Errázuriz Cruz, 2012, 103). Obsérvese (24) para un ejemplo de lo que es introduciendo el tópico.

a. eeh / bueno / yo creo que / confío de alguna manera en lo que son los organismos de control / la DINAMA por ejemplo / [MONV_M13_003] 
Aquí, lo que son precede a la categoría genérica "los organismos de control". Aunque en este caso el reemplazo por en lo que respecta a da como resultado un enunciado inaceptable ( ${ }^{*}$ confío de alguna manera en lo que respecta a los organismos de control), la relación general/particular se pone de manifiesto, ya que el elemento ejemplificador, DINAMA (Dirección Nacional de Medio Ambiente), aparece seguido del MD de concretización más canónico, "por ejemplo".

Esta doble función tematización/concretización, que podría parecer inicialmente contradictoria, ya ha sido observada por Fernández Bernárdez en formas más canónicas de la ejemplificación.

Cuando el ejemplo es un tema [...] el marcador actúa como tematizador [...] Pero cuando el ejemplo no es un tema, el marcador focaliza un segmento del enunciado con la finalidad de concretar o especificar algo que se ha dicho anteriormente (1994-1995, 127-128).

Otro estudio sobre operadores de concreción en el español hablado de Caracas encuentra que, en 50 de los 242 casos analizados de por ejemplo, el elemento de concreción posee una función topicalizadora (González Díaz, 2013, 75).

Hay un tercer tipo de función mencionada por Lastra y Butragueño (2016). Ellos hacen notar que las PIR, aún siendo focales, "[s]uelen presentarse sin contraste [...] pero cuando lo presentan suelen recurrir a elementos espacio-temporales" (281). A continuación, un ejemplo de los autores que presenta contraste espacial y uno de mi cuerpo de datos con contraste temporal. 
me subo al metro y hasta Pantitlán [...] sí/ pues <-ps> se va casi todo $l o$ que es Circuito Interior. (Lastra y Butragueño, 2016, 277)

no todo el año no // depende de cuando arrancan [a ensayar las comparsas] junio agosto todas eso / todas esas fechas / ahora más porque estamos más cerca de lo que es el carnaval [MONV_H11_035]

Esta función contrastiva, nuevamente, no se aleja del comportamiento normal de los MD de ejemplificación: González Díaz (2013) ha cuantificado, solo para por ejemplo, 27 usos contrastivos de 242 casos estudiados.

Por otra parte, lo que sucede, tanto en (25) como en (26), es que la construcción lo que es recorta una porción menor dentro de un marco espacial o temporal mayor, o bien destaca el marco frente a su porción. En (25), por ejemplo, Pantitlán es una estación de metro en la Ciudad de México, desde la cual se puede observar el Circuito Interior Bicentenario, que es una vía de circunvalación que rodea el primer cuadro de la capital mexicana. En (26) lo que es destaca un período temporal específico (el carnaval) en el que las comparsas ensayan. Este período se recorta dentro de un marco temporal más general, “todo el año”. En (27), se observa un caso de contraste espacial en el que, a diferencia de (25), lo que es precede a la porción y no al dominio mayor: "la escuela" está contenida dentro de "barrio". 
el barrio me encantó / pero lo que era la escuela no [MONV_H21_043]

Por último, algunos de los casos de lo que es aparecen sin un elemento generalizador aparente, como en (28).

Les enseñan más de chico a lo que es números / letras todas esas cosas / entonces / a la actividades que hacen /escriben dibujan todo / van aprendiendo más [MONV_H11_035]

Estos casos pueden quedar también comprendidos dentro del universo de la ejemplificación. Fernández Bernárdez ha dicho que en las situaciones en las que hay un ejemplo pero no hay un término general hay una presuposición pragmática: “asumimos un término general al que se ejemplifica” (19941995, 116).

\section{Reflexiones finales: Gramática de la Aplicación}

Como se pudo observar, la hipótesis del MD de concreción comparte un gran número de rasgos con la de las PIR? . El Cuadro 2 expone los rasgos

9 Hay tres rasgos enunciados por Lastra y Butragueño (2016) para las PIR que no comparto, como se desprende del análisis expuesto. Los primeros dos son el alcance oracional de la construcción (e) la explicitud del correferente y la relación predicativa entre lo que es y el consecuente (d): sostener que lo que es es un MD presupone, necesariamente, que el alcance es extraoracional; por la misma 


\section{asignados por Lastra y Butragueño a las PIR (Lastra y Butragueño, 2016) y cuáles de ellos son compartidos por los MDs en general o por los MDs de concreción en particular.}

Cuadro 2. Comparación de los rasgos propuestos por Lastra y Butragueño (2016) con los rasgos de los MDs en general y de los MDs de concreción en particular

\begin{tabular}{|l|l|}
\hline Rasgos de las PIR & Rasgos de los MDs \\
\hline $\begin{array}{l}\text { La invariabilidad de lo que. La } \\
\text { concordancia no categórica entre el verbo } \\
\text { débil y el consecuente. La tendencia al } \\
\text { presente en el verbo débil }\end{array}$ & $\begin{array}{l}\text { Los MD son unidades invariables. Se } \\
\text { admite, no obstante, que no todos los } \\
\text { MDs están igualmente gramaticalizados } \\
\text { (Martín Zorraquino y Portolés Lázaro, } \\
1999,4060) .\end{array}$ \\
\hline $\begin{array}{l}\text { El carácter no extraíble del consecuente, } \\
\text { es decir, que el consecuente no puede ser } \\
\text { destacado por una PdR }\end{array}$ & $\begin{array}{l}\text { Los MDs no se pueden destacar por } \\
\text { medio de una PdR por tratarse de } \\
\text { unidades no integradas en la oración } \\
\text { (Martín Zorraquino y Portolés Lázaro, } \\
1999,4067) .\end{array}$ \\
\hline $\begin{array}{l}\text { El papel de la negación: es posible en la } \\
\text { oración principal pero no dentro de la PIR }\end{array}$ & $\begin{array}{l}\text { Los MDs no pueden ser negados (Martín } \\
\text { 4067). }\end{array}$ \\
\hline
\end{tabular}

razón, lo que es no puede establecer una relación predicativa (oracional) con el consecuente. El tercer rasgo es la naturaleza referencial de lo (inciso [c]) puesto que, desde la perspectiva del MD, lo ha perdido sus rasgos sintáctico-semánticos en favor de la función pragmalingüística. Por otra parte, no he confrontado los MDs de concreción con las características de las PIR propuestas por Lastra y Butragueño que no han sido mencionadas por la literatura sobre los MDs en general ni sobre los MD de concreción en particular; estos son: la modulación de la fuerza ilocutiva por parte de algunas PIR (k) y el carácter objetival del correferente (b). Sobre el segundo, sí he dado con ejemplos (ver [7] supra) que lo contradicen. No descarto, empero, que se pueda dar cuenta de ellas desde la mirada aquí propuesta. En tercer lugar, no he considerado los rasgos negativos, como el inciso (i) (la improbabilidad de que la construcción reciba prominencia prosódica) y el (l) (naturaleza no epistémica y no evidencial de las PIR). 
Cuadro 2. (Cont.) Comparación de los rasgos propuestos por Lastra y Butragueño (2016) con los rasgos de los MDs en general y de los MDs de concreción en particular

\begin{tabular}{|l|l|}
\hline Rasgos de las PIR & Rasgos de los MDs de concreción \\
\hline $\begin{array}{l}\text { Posición consecuente del } \\
\text { correferente y carácter de frase } \\
\text { nominal }\end{array}$ & $\begin{array}{l}\text { Sobre como, Fernández Bernárdez dice que "precede } \\
\text { siempre al segmento ejemplificador: carece de } \\
\text { movilidad. Además, el ejemplo suele estar formulado } \\
\text { como un sintagma nominal" (Fernández Bernárdez, } \\
\text { 1994-1995, 121). }\end{array}$ \\
\hline $\begin{array}{l}\text { El carácter comúnmente } \\
\text { aseverativo de los actos de } \\
\text { habla en los que se instalan las } \\
\text { PIR }\end{array}$ & $\begin{array}{l}\text { Fuentes Rodríguez estableció que los marcadores } \\
\text { de ejemplificación se distinguen de acuerdo con } \\
\text { los diferentes tipos de modalidad oracional que } \\
\text { permiten: por ejemplo puede combinarse con } \\
\text { cualquier modalidad, frente a asi que se utiliza con } \\
\text { más frecuencia con declarativas (Fuentes Rodríguez, } \\
1987,190) .\end{array}$ \\
\hline $\begin{array}{l}\text { La existencia de PIR } \\
\text { prototípicas y PIR periféricas: } \\
\text { las PIR casi siempre } \\
\text { son prescindibles (PIR } \\
\text { prototípicas), en contraste } \\
\text { con las que no cumplen con } \\
\text { criterios de eliminabilidad (PIR } \\
\text { periféricas) }\end{array}$ & $\begin{array}{l}\text { Fernández Bernárdez sostiene que la elisión de una } \\
\text { forma de ejemplificación puede indicar que los } \\
\text { casos expuestos son los únicos ejemplos posibles; } \\
\text { la ausencia también puede suponer la imposición } \\
\text { brusca de un tema en lugar de una propuesta; en } \\
\text { tercer lugar, la presencia/ausencia a veces determina la } \\
\text { oposición de «ejemplificación» frente a adición, entre } \\
\text { 113-115). }\end{array}$ \\
\hline $\begin{array}{l}\text { La función informativa de } \\
\text { las PIR: refieren al foco en el } \\
88.2 \% \text { de los casos, pero que } \\
\text { también pueden "desempenar } \\
\text { cualquier función informativa" } \\
\text { (2016: 275) }\end{array}$ & $\begin{array}{l}\text { González Díaz encontró que 100 de los 242 casos } \\
\text { analizados de por ejemplo presentan un valor } \\
\text { focalizador y que en 50 de los 242, el elemento } \\
\text { de concreción posee una función topicalizadora/ } \\
\text { tematizadora (González Díaz, 2013, 75). }\end{array}$ \\
\hline $\begin{array}{l}\text { La equivalencia entre las PIR y } \\
\text { se que tiene que ver con o lo que a, sugerida por Lastra } \\
\text { y Butragueño en el inciso (h) }\end{array}$ & $\begin{array}{l}\text { Esto pone de manifiesto una gran relación semántica } \\
\text { de las PIR con los MDs de topicalización. Ya } \\
\text { hemos hablado de la doble función de los MDs de } \\
\text { ejemplificación. }\end{array}$ \\
\hline
\end{tabular}


Cuadro 2. (Cont.) Comparación de los rasgos propuestos por Lastra y Butragueño (2016) con los rasgos de los MDs en general y de los MDs de concreción en particular

\begin{tabular}{|l|l|}
\hline Rasgos de las PIR & Rasgos de los MDs de concreción \\
\hline $\begin{array}{l}\text { El papel de la cuantificación: } \\
\text { cierto número de casos } \\
\text { presentan un cuantificador, casi } \\
\text { siempre todo. }\end{array}$ & $\begin{array}{l}\text { Si bien no he encontrado ejemplos de cuantificación } \\
\text { en marcadores de concreción, sí existe en } \\
\text { construcciones como en lo que respecta a, lo que tiene } \\
\text { que ver con, etc. }\end{array}$ \\
\hline $\begin{array}{l}\text { La naturaleza habitualmente no } \\
\text { contrastiva de las PIR. Cuando } \\
\text { presenta contraste, suelen } \\
\text { recurrir a elementos espacio- } \\
\text { temporales. }\end{array}$ & $\begin{array}{l}\text { González Díaz ha cuantificado, solo para por } \\
\text { ejemplo, 27 usos contrastivos de } 242 \text { casos estudiados } \\
\text { (González Díaz, 2013, 75). }\end{array}$ \\
\hline
\end{tabular}

Dado el alto grado de coincidencia entre ambas descripciones, quisiera, en último lugar, hacer una breve reflexión sobre por qué esta diferencia va más allá de un problema ontológico y se constituye como un problema para la aplicación.

Como dijimos, para Corder no existe una sola gramática observacionalmente adecuada (Corder, 1982 [1981], 59). Un estudio empírico como el trabajo de Lastra y Butragueño ya mencionado es observacionalmente adecuado: se describe la construcción mediante rasgos advertibles y mensurables. El problema comenzará en el momento en que se quiera aplicar el contenido en la enseñanza ELE, pues ¿cómo pedagogizar los 14 incisos propuestos para las PIR? O bien, ¿`cómo hacer de la descripción genérica de la función (las PIR refieren al foco) una descripción ELE operativa? Entender lo que es como un marcador de concreción vuelve la construcción más “enseñable”. 
A esta búsqueda teórica de una explicación que no sea solo empírica, sino que tenga su atención crítica puesta al servicio de la aplicación es a lo que he dado en llamar Gramática de la Aplicación.

\section{CONCLUSIONES}

Se ha demostrado, para este corpus, que la construcción lo que es se comporta como un MD, más específicamente un marcador de concreción.

Hasta el momento, la literatura ha partido de la premisa de que lo que es es una PdR y se la ha comparado con otras construcciones con lo que. El trabajo más minucioso les corresponde a Lastra y Butragueño (2016), quienes describen este fenómeno, que han llamado PIR, detallando 14 características que, en ocasiones, también son compartidas por otras estructuras con $l o$ que. Sin embargo, son 9 los atributos que las PIR no comparten con las PdR: En las PdR, el pronombre relativo no es invariable como en las PIR; en las PdR, el elemento escindido no es de carácter predominantemente objetival; en las PdR, el constituyente escindido es extraíble y en las PIR no; en las PdR, el pronombre relativo no es equivalente a tener que ver con, etc.; las PdR presentan mayor concordancia que las PIR; las PdR no presentan una tendencia al presente como las PIR; las PdR no son cuantificables; las PdR presumiblemente no presentan una distribución social como las PIR; ninguna de las variables de las PdR propone el verbo cópula inmediatamente después del relativo. 
$\mathrm{Al}$ mismo tiempo, lo que es se asemeja a los MDs en general y a los de concreción en particular: Los MDs también presentan diferentes grados de gramaticalización, no pueden ser destacados por una PdR ni ser negados, hay MDs de concreción cuyo correferente aparece en posición pospuesta y es de carácter nominal, hay MDs de concreción que ocurren comúnmente en enunciados aseverativos y hay casos en los que la marca de ejemplificación puede ser elidida, generando cambios en el significado; los MDs de concreción, aunque suelen referirse al foco, también pueden ser topicalizadores y ser de naturaleza contrastiva y no contrastiva. Puesto que lo que es se ajusta, en general, a las propiedades establecidas para los MDs, considero pertinente incluir la forma en esta categoría.

Por último, concluir que lo que es no es una $\mathrm{PdR}$ sino un $\mathrm{MD}$ no es simplemente un desacuerdo ontológico; es una búsqueda teórica de una explicación que, además de empírica, esté al servicio de la aplicación y que he llamado Gramática de la Aplicación.

Será un trabajo para el futuro, pues, comparar lo que es con otros marcadores discursivos de concreción para analizar qué es lo propio de esta forma en oposición a otros marcadores de concreción/ejemplificación, entender por qué es una forma útil para reforzar contraste, determinar marcas sociolingüísticas, etc.

\section{ReEERENCIAS BibLIOGRÁFICAS}

Bourdieu, Pierre; Chamboredon, Jean-Claude; Passeron, Jean-Claude. El oficio de sociólogo. Buenos Aires: Siglo XXI ed., 2002(1975). 
Carbonero Cano, Pedro. "Las Construcciones con 'lo que' y su Uso en el Habla de Sevilla”. In: Sociolingüistica Andaluza 12. Identidad Lingüistica y Comportamientos Discursivos, 1, 2001, 63-98.

Castañeda, Alejandro; Melguizo Moreno, Elisabeth. "Querían dormirlo, se ha dormido, está durmiendo. Gramática cognitiva para la presentación de los usos del se en clase de ELE”. In: Mosaico, 18, 2006, 13-20.

Cifuentes Honrubia, José L. "Bases sintácticas y bases semánticas de la inacusatividad en verbos de movimiento". In: Revista de investigación lingüistica, 2(2), 1999, 37-72.

Corder, Stephen P. Error analysis and interlanguage. Oxford: Oxford University Press, 1982(1981).

Errázuriz Cruz, María C. "Análisis del uso de los marcadores discursivos en argumentaciones escritas por estudiantes universitarios". In: Perfiles educativos, 34(136), 2012, 98-117. Disponible en: <http://www.scielo.org.mx/scielo. php?script=sci_arttext\&pid=S0185-26982012000200007\&lng=es\&tlng=es $>$. Consultado el 1 de julio de 2019.

Fernández Bernárdez, Cristina. "Marcadores textuales de 'ejemplificación' textual". In: E.L.U.A., 10, 1994-1995, 103-144.

Fuentes Rodríguez, Catalina. Enlaces extraoracionales. Sevilla: Alfar, 1987.

Fundéu BBVA. "Lo que es, construcción innecesaria". Disponible en: <www.fundeu. es/recomendacion/lo-que-es-construccion-innecesaria-1070>. Consultado el 1 de junio de 2019.

González Díaz, Consuelo. "Uso de por ejemplo, por lo menos, de repente y otros operadores de concreción en el español hablado de caracas". In: Boletín de Lingüistica, 25(39-40), ene-dic 2013, 61-91.

Lastra, Yolanda; Butragueño, Pedro M. "Allá llega a lo que es el pueblo de San Agustín: El caso de la perífrasis informativa con lo que es en 'el corpus sociolingüístico de la Ciudad de México'”. In: Lingüistica y Literatura, 69, 2016, 269-293.

Martín Sánchez, Miguel A. "Apuntes a la historia de la enseñanza de lenguas extranjeras: la enseñanza de la gramática”. In: Tejuelo, 8, 2010, 59-76. 
Martín Zorraquino, María A.; Portolés Lázaro, José. "Los marcadores del discurso". In: Bosque, Ignacio; Demonte, Violeta (eds.). Nueva Gramática Descriptiva de la Lengua Española. Madrid: Espasa-Calpe, 1999, 4215-4244.

Moreno Cabrera, Juan C. "Las funciones informativas. Las perífrasis de relativo y otras construcciones perifrásticas". In: Bosque, Ignacio; Demonte, Violeta (eds.). Nueva Gramática Descriptiva de la Lengua Española. Madrid: EspasaCalpe, 1999, 4245-4302.

Pérez Álvarez, Bernardo E. "Funciones oracionales y discursivas de la construcción lo que". In: Bogard Sierra, Sergio (ed.). Del léxico al discurso. La construcción gramatical del sentido en español. México: El Colegio de México, 2014, 71-98. 